\title{
Expression of Microphthalmia Transcription Factor in Sentinel Lymph Nodes of Patients with Melanoma
}

\author{
Minia Hellan, Michelle S. Gentile, Luay Ailabouni, George I. Salti*
}

Department of Surgical Oncology, University of Illinois at Chicago, Chicago, USA.

Email: geosalti@uic.edu

Received January $11^{\text {th }}, 2011$; revised May 20 $0^{\text {th }}$ 2011; accepted May 30 $0^{\text {th }}, 2011$.

\begin{abstract}
Background: Sentinel lymph node biopsy is widely used in the management of melanoma patients. Multiple markers are used to stain sentinel lymph node tissue including S100, HMB-45 and melan A with different success. We investigated, for the first time, the use of Microphthalmia transcription factor (Mitf) staining in a larger series of sentinel lymph nodes. Mitf is a transcription factor essential for the development and survival of melanocytes. It has been introduced recently as a sensitive and specific marker for melanomas. Methods: Thirty patients with cutaneous melanoma were included in our study: twenty patients underwent sentinel lymph node biopsy; ten patients underwent complete lymph node dissection for clinically positive disease. Results: Ten out of twenty sentinel lymph nodes were negative for tumor cells and showed no Mitf staining. Out of the ten positive sentinel lymph nodes, eight were also positive for Mitf. Only four out of the ten clinically positive lymph nodes stained for Mitf. Conclusions: We conclude that Mitf can be used as an additional marker for evaluating sentinel lymph nodes in patients with melanoma. In addition, our results imply that Mitf is involved in melanoma differentiation.
\end{abstract}

Keywords: Mitf, Melanoma, Sentinel Lymph Node

\section{Introduction}

Trends in the management of patients with malignant melanoma are rapidly changing. Novel techniques for lymphatic mapping of regional lymph nodes with sentinel lymphadenectomy have greatly impacted the care of these patients eliminating the need for elective lymphadenectomy in approximately $80 \%$ of cases [1]. Pathogic sentinel lymph node (SLN) status in clinically nodeegative patients is an important independent prognostic factor $[2,3,4]$. The number of metastatic nodes, the tumor burden at the time of staging, and the presence or absence of ulceration of the primary melanoma are the most significant predictors of outcome as shown in an multivariate analysis of 17,600 melanoma patients $[3,4]$. However, the presence of melanoma cells in SLNs can be difficult to detect, even when sections from the entire node are cut and examined thoroughly. All lymph nodes other than those that contain obvious tumor on visual inspection must be examined by immunohistochemistry. If immunohistochemistry is not routinely performed, approximately $12 \%$ of positive SLNs will be miscatego- rized as tumor negative [5]. Traditionally used immunhistochemical markers in melanoma have disadvantages. Monoclonal antibody to S100 protein, a calcium-binding protein originally isolated from cow brain, is a sensitive marker that reacts with more than $90 \%$ of melanomas. It remains the most sensitive marker for both neval cells and melanomas, including amelanotic and spindle cell variants, but unfortunately, cannot differentiate benign from malignant cells [5-8]. In addition, S100 lacks sufficient specificity and reacts with a wide range of tissues including dentritic cells, Schwann cells, adipocytes, chondrocytes and others [5-8]. The HMB-45 antibody recognizes the melanosome-specific glycoprotein gp100 and is specificity is described as high as $97 \%$ for detecting melanoma [6]. However, HMB-45 is detectable in only $50 \%$ to $75 \%$ of all melanomas $[5,6,8]$. Melan A protein is a melanocytic differentiation antigen and a product of the MART-1 gene. It is a new antibody which stains both benign and malignant melanocytic lesions in a very similar fashion to that of S100 and seems to be more reliable than HMB-45 [8]. 
Microphthalmia transcription factor (Mitf) has recently been introduced as the only nuclear melanocytic marker [9-11]. Mitf is a transcriptional regulator and a nuclear component of the signaling transduction pathway [12]. It is important for the survival of melanocytes and regulates the expression of several melanocyte genes. Multiple studies have confirmed that Mitf is a sensitive marker for epitheloid melanomas: $82 \%$ to $100 \%$ of melanomas are positive for Mitf [6,9,10,11,13,14]. Recently we suggested that Mitf may be a new molecular prognostic marker in melanoma patients with low levels of Mitf expression correlating with worse prognosis [9]. The use of Mitf staining in sentinel lymph nodes has only been investigated in a very small series of four sentinel lymph nodes up to date [15]. The purpose of the present study was to further evaluate the usefulness of Mitf staining in the evaluation of SLNs in patients with melanoma.

\section{Patients and Methods}

\subsection{Patients}

We randomly selected 30 patients from the tumor registry of the Department of Surgical Oncology at the University of Illinois in Chicago Medical Center. Twenty patients with primary melanomas greater that one millimeter thick and clinically negative lymph node basins underwent sentinel lymph node biopsy following injection of radio labeled colloid and isosulfan blue dye. An additional ten patients with clinically positive lymph nodes underwent complete lymph node dissection. All SLNs and clinically positive nodes were evaluated for Mitf staining by immunohistochemistry.

\subsection{Histopathologic Examination of Lymph Node Specimens}

Standard technique for preparation of SLNs was followed as previously described [16]. Briefly, the excised lymph nodes were examined in a routine fashion with visual inspection to detect tumor nodules. The nodes were bisected from hilum to periphery and then fixed in $10 \%$ neutral buffered formalin, embedded in paraffin and sectioned. The nodal sections were then processed for routine hematoxylin and eosin (H\&E) staining. Lymph nodes negative for metastasis on $\mathrm{H} \& \mathrm{E}$ staining were also processed for peroxidase immunostaining using antibodies to HMB-45 or melan-A protein (Dako, Carpinteria, CA, USA). Both negative and positive control stains were run simultaneously with the specimens to confirm the sensitivity and specificity of the immunhistochemical method. SLNs were level-sectioned for routine H\&E three times, for HMB-45 and melan-A immunohistochemistry two times. All lymph nodes were processed for Mitf staining: Standard avidin-biotin-peroxidase immu- nohistochemistry was performed with the mouse monoclonal IgG1 micropthalmia antibody D5 (Neomarkers, Union City, CA, USA). Antigen retrieval was accomplished with 1mM EDTA buffer. We used 1\% dried skim milk to block nonspecific binding before incubation with the primary antibody for two hours (1:25 dilution). Aminoethylcarbazine was used as a chromagen [9].

\section{Results}

Of the twenty SLNs, ten had metastatic disease noted by H\&E staining. The remaining ten SLNs did not show evidence of metastatic disease either by H\&E or by immunohistochemistry. All ten negative sentinel lymph nodes did not express Mitf. Of the positive SLNs eight (80\%) stained for Mitf by immunohistochemistry. These SLNs were not stained for HMB-45 or Melan A and thus direct comparison with Mitf staining is not feasible. All ten clinically-positive lymph nodes showed evidence for metastatic disease by H\&E staining. Only four (40\%) stained positive for Mitf. These results are summarized in Table 1. Nuclear staining for Mitf is shown in Figure 1.

\section{Discussion}

Mitf, a bHLHZip (basic/helix-loop-helix/leucine zipper) transcription factor, is essential for the development and maintenance of the melanocyte lineage [12,17]. It can both activate and repress transcription. Several pigment cell-specific genes, including the tyrosinase (Tyr) and tyrosinase-related genes, TRP-1 and DCT/TRP-2 are regulated by Mitf [12,18]. In mice, the allele Mitf mi leads to complete absence of melanocytes in the homozygous state [12]. In contrast to various melanocytic markers, such as melanin or c-kit, whose expression may be lost or difficult to detect in malignant melanocytic lesions, Mitf expression is usually maintained in human melanoma specimens $[7,9,10,11,13,14]$. Different signaling molecules or transcription factors implicated genetically in melanocyte development are known to either affect Mitf expression or its function [19]. Mitf has emerged as a potentially important diagnostic marker for melanoma. King et al reported that Mitf is a sensitive and specific melanocyte marker for melanoma diagnosis [10]. They showed $100 \%$ nuclear staining for 76 primary melanomas using the monoclonal antibody D5 and in none of 60 non-melanoma tumors. In a follow-up study

Table 1. Nuclear staining for Mitf.

\begin{tabular}{ll}
\hline Lymph node status & Mitf staining $^{\mathbf{a}}$ \\
\hline Negative SLNs $^{\mathrm{b}}$ & 0 \\
Positive SLNs & 8 \\
Clinically-positive lymph nodes & 4 \\
\hline
\end{tabular}

a: 10 patients evaluated in each category. b: SLNs = sentinel lymph nodes. 


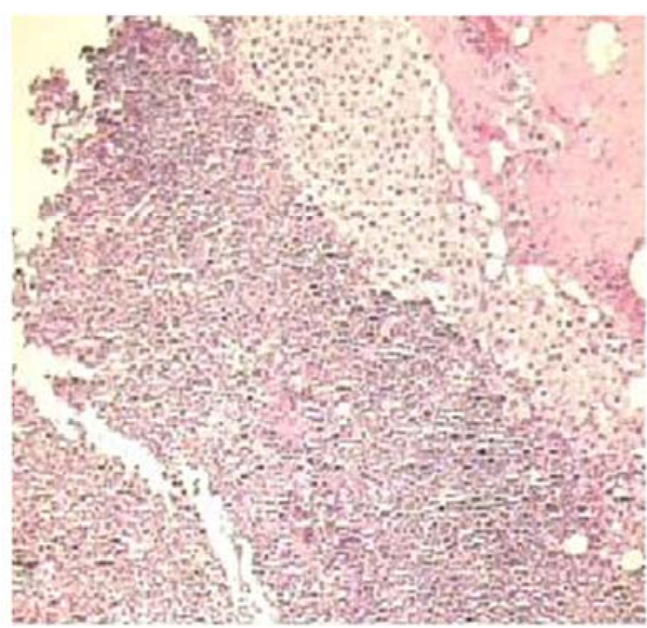

(a)

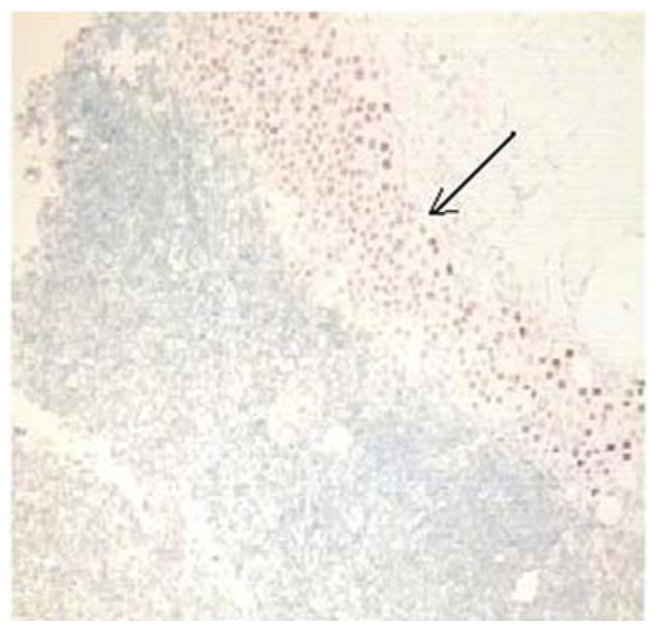

(b)

Figure 1. Positive sentinel lymph node of a melanoma patient, original magnification $\times 10$. (a): H\&E staining of lymph nodes showing metastatic melanoma. (b): Note nuclear staining of Mitf (arrow).

the authors demonstrated uniform conservation of Mitf antibody expression in both benign and malignant melanocytic lesion with the exception of desmoplastic malignant melanomas $[7,11]$.

We previously reported positive Mitf staining in 83\% of patients with intermediate thickness melanomas [9]. In the years following these initial studies, a multitude of manuscripts were published describing the utility of Mitf antibodies for the diagnosis of various types of melanomas and other cancers. Antibody-detected Mitf expression was also found in $100 \%$ of benign nevi and $100 \%$ of primary cutaneous melanomas with the exception of desmoplastic melanomas (1/14) [11]. The literature on the immunoreactivity of Mitf in melanocytic and non melanocytic spindle cell lesions remains confusing. Koch et al reported that 55\% (11/20) of spindle cell and desmoplastic melanomas were immunoreactive for Mitf, but none of 12 malignant peripheral nerve tumors and only 2 of 10 neurofibromas were positive giving Mitf a higher sensitivity and specificity than that of HMB-45 [20]. Other investigators however were unable to confirm these findings. In 2 studies, one third to one half of nonmelanocytic spindle cell tumors and only $30 \%$ of spindle cell and desmoplastic melanomas were positive for Mitf [21,22]. In addition $8 \%$ of 386 non-melanocytic tumors stained positive for Mitf including macrophages, fibroblasts, Schwann cells and smooth muscle cells [22]. On the other hand the nuclear staining of Mitf can allow for clearer distinction from pigmented cytoplasm, often a confounding issue during interpretation [15].

In our study, none of the 10 negative SLNs were upstaged with Mitf staining. In this small number of SLNs, Mitf demonstrates a specificity of $100 \%$. This is comparable to a specificity of $97 \%$ as described by Sheffield et al. in 72 primary melanomas and 32 nonmelanocytic malignancies [6] and a specificity of $100 \%$ as found by Dorvault et al. [14]. Miettinen et al. suggest caution in the use of Mitf in the search for nodal micrometastasis as they found occasional Mitf-positive nuclei in histiocytes of germinal centers in 8 out of 20 lymph nodes and in sinus histiocytes in 7 out of 22 lymph nodes. Interpreting Mitf-positive histiocytes as melanoma cells should be avoided [7]. We did not encounter this problem in our 10 negative SLNs. Our study shows that $80 \%$ of SLNs which are positive by H\&E staining also stain for Mitf by immunohistochemistry. In a small serious of only 4 sentinel lymph nodes positive of metastatic melanoma strong nuclear staining was observed in all 4 lymph nodes [15]. Interestingly, only $40 \%$ of clinically-positive lymph nodes (all of which show metastatic melanoma by H\&E) stain for Mitf. This is in contrast to the results described by Miettinen et al, who evaluated Mitf staining in 266 metastatic melanomas including 130 patients with lymph node metastases and found Mitf staining in $88 \%$ of these tumors [7]. It must be noted that in their study, an antibody cocktail of C5 + D5 was used, whereas we used pure D5 monoclonal Mitf antibody.

We propose that levels of Mitf expression imply a more differentiated melanoma and correlate with prognosis. In our previous study we found that mean overall survival and disease-free survival in patients whose melanomas did not express Mitf were $80+/-18$ months and $71+/-20$ months respectively. This compared with $187+/-13$ and $186+/-14$ months for patients whose melanomas expressed Mitf [9]. In addition, the pathologic status of the SLN remains the most important prognostic factor in patients with cutaneous melanoma $[3,23]$. The revised melanoma staging system concludes 
that the number of metastatic lymph nodes and the "tumor burden" were the most dominant predictors of survival in patients with Stage III melanoma [3,4]. Patients with metastatic nodes detected by palpation had a shorter survival compared with patients whose nodal metastases were first detected by sentinel node biopsy. Taken together, the decreased Mitf staining in the macroscopically positive lymph nodes implies that Mitf is a differentiation marker, whose loss of expression correlates with advanced disease and poor prognosis. In other words, higher levels of antigen expression represent a more differentiated state, while lower levels represent a less differentiated state. Interestingly data supporting this hypothesis were reported in vitro and in a SCID mice model by Selzer et al. [24]. The authors nicely demonstrated that transfection of the melanocyte specific isoform of Mitf (Mitf-M) into a melanoma cell line (518A2) lacking the M-isoform and into a permanent cell line established from normal melanocytes (NMEL-II) resulted in slower tumor growth. In addition to the growthinhibitory effects, Mitf-M expression led to a change in the histopathological appearance of tumors from epitheloid toward a spindle-cell type in vivo. Similarly, we have shown that Mitf-M transfected into the aggressive UISO-Mel-6 melanoma cell lines abrogated liver and lung metastases and upregulated the markers of differentiation [25].

\section{Conclusions}

Our results show that Mitf can be used as an additional marker for evaluating sentinel lymph nodes in patients with melanoma. To confirm these preliminary results in a relative small number of patients, larger studies will be necessary. In addition, Mitf appears to be involved in melanoma differentiation and leads to a less aggressive phenotype.

\section{REFERENCES}

[1] M. C. Kelley, D. W. Ollila and D. L. Morton, "Lymphatic Mapping and Sentinel Lymphadenectomy for Melanoma," Seminars in Surgical Oncology, Vol. 14, No. 4, 1998, pp. 283-290.

doi:10.1002/(SICI)1098-2388(199806)14:4<283::AID-SS $\mathrm{U} 4>3.0 . \mathrm{CO} ; 2-\mathrm{Z}$

[2] J. E. Gershenwald, W. Thompson, P. F. Mansfield, J. E. Lee, M. I. Colome, C. H. Tseng, et al., "Multi-Institutional Melanoma Lymphatic Mapping Experience: The Prognostic Value of Sentinel Lymph Node Status in 612 Stage I or II Melanoma Patients,” Journal of Clinical Oncology, Vol. 17, No. 3, 1999, pp. 976-983.

[3] C. M. Balch, S. J. Soong, J. E. Gershenwald, J. F. Thompson, D. S. Reintgen, N. Cascinelli, et al. "Prognostic Factors Analysis of 17,600 Melanoma Patients: Validation of the American Joint Committee on Cancer Mela- noma Staging System,” Journal of Clinical Oncology, Vol. 19, No. 16, 2001, pp. 3622-3634.

[4] C. M. Balch, S. J. Soong, M. B. Atkins, A. C. Buzaid, N. Cascinelli, D. G. Coit et al. "An Evidence-Based Staging System for Cutaneous Melanoma,” CA: A Cancer Journal for Clinicians, Vol. 54, No. 3, 2004, pp. 131-149. doi:10.3322/canjclin.54.3.131

[5] A. J. Cochran, B. R. Balda, H. Starz, D. Bachter, D. N. Krag, C. W. Cruse, et al., "The Augsburg Consensus. Techniques of Lymphatic Mapping, Sentinel Lymphadenectomy, and Completion Lymphadenectomy in Cutaneous Malignancies,” Cancer, Vol. 89, No. 2, 2000, pp. 236-241.

doi:10.1002/1097-0142(20000715)89:2<236::AID-CNCR 5>3.0.CO;2-0

[6] M. V. Sheffield, H. Yee, C. C. Dorvault, K. N. Weilbaecher, I. A. Eltoum, G. P. Siegal et al. "Comparison of Five Antibodies as Markers in the Diagnosis of Melanoma in Cytologic Preparations," American Journal of Clinical Pathology, Vol. 118, No. 6, 2002, pp. 930-936. doi:10.1309/EWK9-LUPR-6BC5-1GXV

[7] M. Miettinen, M. Fernandez, K. Franssila, Z. Gatalica, J. Lasota and M. Sarlomo-Rikala, "Microphthalmia Transcription Factor in the Immunohistochemical Diagnosis of Metastatic Melanoma: Comparison with Four Other Melanoma Markers," The American Journal of Surgical Pathology, Vol. 25, No. 2, 2001, pp. 205-211. doi:10.1097/00000478-200102000-00008

[8] K. Blessing, D. S. Sanders and J. J. Grant, “Comparison of Immunohistochemical Staining of the Novel Antibody Melan-A with S100 Protein and HMB-45 in Malignant Melanoma and Melanoma Variants," Histopathology, Vol. 32, No. 2, 1998, pp. 139-146. doi:10.1046/j.1365-2559.1998.00312.x

[9] G. I. Salti, T. Manougian, M. Farolan, A. Shilkaitis, D. Majumdar and T. K. Das Gupta, "Micropthalmia Transcription Factor: A New Prognostic Marker in Intermediate-Thickness Cutaneous Malignant Melanoma," Cancer Research, Vol. 60, No. 18, 2000, pp. 5012-5016.

[10] R. King, K. N. Weilbaecher, G. McGill, E. Cooley, M. Mihm and D. E. Fisher, "Microphthalmia Transcription Factor. A Sensitive and Specific Melanocyte Marker for Melanoma Diagnosis," American Journal of Pathology, Vol. 155, No. 3, 1999, pp. 731-738. doi:10.1016/S0002-9440(10)65172-3

[11] R. King, P. B. Googe, K. N. Weilbaecher, M. C. Mihm Jr and D. E. Fisher, "Microphthalmia transcription factor expression in cutaneous benign, malignant melanocytic, and nonmelanocytic tumors," The American Journal of Surgical Pathology, Vol. 25, No. 1, 2001, pp. 51-57. doi:10.1097/00000478-200101000-00005

[12] E. Steingrimsson, N. G. Copeland and N. A. Jenkins, "Melanocytes and the Microphthalmia Transcription Factor Network," Annual Review of Genetics, Vol. 38, 2004, pp. 365-411.

[13] S. R. Granter, K. N. Weilbaecher, C. Quigley and D. E. Fisher, "Role for Microphthalmia Transcription Factor in 
the Diagnosis of Metastatic Malignant Melanoma,” Applied Immunohistochemistry \& Molecular Morphology, Vol. 10, No. 1, 2002, pp. 47-51. doi:10.1097/00022744-200203000-00008

[14] C. C. Dorvault, K. N. Weilbaecher, H. Yee, D. E. Fisher, L. A. Chiriboga, Y. Xu and D. C. Chhieng, "Microphthalmia Transcription Factor: A Sensitive and Specific Marker for Malignant Melanoma in Cytologic Specimens," Cancer, Vol. 93, No. 5, 2001, pp. 337-343. doi:10.1002/cncr.9049

[15] F. M. O’Reilly, D. J. Brat, B. E. McAlpine, H. E. Grossniklaus, A. L. Folpe and J. L. Arbiser, "Microphthalmia Transcription Factor Immunohistochemistry: A Useful Diagnostic Marker in the Diagnosis and Detection of $\mathrm{Cu}-$ taneous Melanoma, Sentinel Lymph Node Metastases, and Extracutaneous Melanocytic Neoplasms," Journal of the American Academy of Dermatology, Vol. 45, No. 3, 2001, pp. 414-419. doi:10.1067/mjd.2001.117526

[16] I. A. Jacobs, C. K. Chang, T. K. Das Gupta and G. I. Salti, "Role of Sentinel Lymph Node Biopsy in Patients with Thin $(<1 \mathrm{~mm})$ Primary Melanoma," Annals of Surgical Oncology, Vol. 10, No. 5, 2003, pp. 558-561. doi:10.1245/ASO.2003.10.025

[17] C. A. Hodgkinson, K. J. Moore, A. Nakayama, E. Steingrimsson, N. G. Copeland, N. A. Jenkins, et al., "Mutations at the Mouse Microphthalmia Locus are Associated with Defects in a Gene Encoding a Novel Basic-HelixLoop-Helix-Zipper Protein,” Cell, Vol. 74, No. 2, 1993, pp. 395-404. doi:10.1016/0092-8674(93)90429-T

[18] C. A. Ferguson and S. H. Kidson, "The Regulation of Tyrosinase Gene Transcription,” Pigment Cell Research, Vol. 10, No. 3, 1997, pp. 127-138. doi:10.1111/j.1600-0749.1997.tb00474.x

[19] C. R. Goding, "Mitf from Neural Crest to Melanoma: Signal Transduction and Transcription in the Melanocyte Lineage," Genes \& Development, Vol. 14, No. 14, 2000, pp. 1712-1728.
[20] M. B. Koch, I. M. Shih, S. W. Weiss and A. L. Folpe, "Microphthalmia Transcription Factor and Melanoma Cell Adhesion Molecule Expression Distinguish Desmoplastic/Spindle Cell Melanoma from Morphologic Mimics," The American Journal of Surgical Pathology, Vol. 25, No. 1, 2001, pp. 58-64. doi:10.1097/00000478-200101000-00006

[21] S. R. Granter, K. N. Weilbaecher, C. Quigley, C. D. Fletcher and D. E. Fisher, "Microphthalmia Transcription Factor: Not a Sensitive or Specific Marker for the Diagnosis of Desmoplastic Melanoma and Spindle Cell (Non-Desmoplastic) Melanoma,” The American Journal of Dermatopathology, Vol. 23, No. 3, 2001, pp. 185-189. doi:10.1097/00000372-200106000-00004

[22] K. J. Busam, K. Iversen, K. C. Coplan and A. A. Jungbluth, "Analysis of Microphthalmia Transcription Factor Expression in Normal Tissues and Tumors, and Comparison of Its Expression with S-100 Protein, gp100, and Tyrosinase in Desmoplastic Malignant Melanoma," The American Journal of Surgical Pathology, Vol. 25, No. 2, 2001, pp. 197-204. doi:10.1097/00000478-200102000-00007

[23] J. M. Ranieri, J. D. Wagner, R. Azuaje, D. Davidson, S. Wenck, J. Fyffe et al. "Prognostic Importance of Lymph Node Tumor Burden in Melanoma Patients Staged by Sentinel Node Biopsy," Annals of Surgical Oncology, Vol. 9, No. 10, 2002, pp. 975-981. doi:10.1007/BF02574515

[24] E. Selzer, V. Wacheck, T. Lucas, E. Heere-Ress, M. Wu, K. $\mathrm{N}$. Weilbaecher et al. "The Melanocyte-Specific Isoform of the Microphthalmia Transcription Factor Affects the Phenotype of Human Melanoma," Cancer Research, Vol. 62, No. 7, 2002, pp. 2098-2103.

[25] F. Lekmine, N. Sethakorn, C. K. Chang, T. K. Das Gupta and G. I. Salti, "Role of Microphthalmia-Associated Transcription Factor (Mitf) in Melanoma Differentiation," Biochemical and Biophysical Research, Vol. 354, No. 3, 16 March 2007, pp. 830-835. doi:10.1016/j.bbrc.2007.01.075 